\title{
Performance of the 12-item WHODAS 2.0 in prodromal Huntington disease
}

\author{
Ji-In Kim ${ }^{1}$, Jeffrey D Long ${ }^{1,2}$, James A Mills ${ }^{1}$, Nancy Downing ${ }^{3}$, Janet K Williams ${ }^{3}$ and Jane S Paulsen ${ }^{\star, 1,4,5}$ \\ the PREDICT-HD Investigators and Coordinators of the Huntington Study Group ${ }^{6}$
}

\begin{abstract}
The Diagnostic and Statistical Manual for Mental Disorders, Fifth Edition (DSM-5), recommends the World Health Organization Disability Assessment Schedule (WHODAS) 2.0 for routine clinical use. We tested the utility of the 12-item WHODAS 2.0 in prodromal Huntington disease. Using data from 726 participants and 630 companions over a 3-year follow-up, linear mixed models were fitted to test (1) baseline and longitudinal differences by progression group; (2) participant and companion differences within each group; and (3) sensitivity of the 12-item WHODAS in comparison to the 36-item WHODAS and the Total Functional Capacity (TFC) score from the Unified Huntington's Disease Rating Scale. Participants showed baseline group differences whereas companions showed baseline and longitudinal group differences. Companions reported worse functional decline over time than participants as the disease progresses. The 12-item WHODAS detected longitudinal change better than the 36-item WHODAS and the TFC in the medium progression group. Results suggest the 12-item WHODAS 2.0 can detect baseline and longitudinal differences in prodromal HD and may be useful in HD clinical trials.
\end{abstract} European Journal of Human Genetics (2015) 23, 1584-1587; doi:10.1038/ejhg.2015.11; published online 4 March 2015

\section{INTRODUCTION}

The Diagnostic and Statistical Manual for Mental Disorders, Fifth Edition (DSM-5), recommends the World Health Organization (WHO) Disability Assessment Schedule (WHODAS) 2.0 as a disability measure for routine clinical use. ${ }^{1}$ There are two versions of the WHODAS 2.0-a full version with 36 items and a short version with 12 items. Previously, we reported that the total score of the 36-item WHODAS 2.0 can detect longitudinal changes in daily function in prodromal (before motor diagnosis) HD, and is better at detecting changes in an earlier stage of HD than the Total Functional Capacity (TFC) from the Unified Huntington's Disease Rating Scale. ${ }^{2}$ The current study investigates the performance of the global score of the 12-item version in a large observational study of people with prodromal HD. The aims of the current study are (i) to test disease progression group differences in baseline values and longitudinal change in a separate analysis of participant and companion ratings on the 12-item WHODAS 2.0; (ii) to compare longitudinal participant and companion ratings on the 12-item version; and (iii) to assess the relative sensitivity of the 12 -item version in comparison with the full version and the TFC, in terms of detecting baseline and longitudinal differences.

\section{MATERIALS AND METHODS}

\section{Participants}

A total of 726 participants and 630 companions were administered the WHODAS 2.0 (starting in 2009) in the Neurobiological Predictors of HD (PREDICT-HD) study ${ }^{3}$ with up to 3.11 years of follow-up. On the basis of the HD gene-expansion test results, individuals were classified as cases, if the cytosine-adenine-guanine (CAG) expansion $\geq 36$ or controls, if $\mathrm{CAG}<36$. Cases were classified into three progression groups based on their CAG-Age Product (CAP) capturing different disease progression levels at baseline. ${ }^{4}$ For the low, medium, and high CAP groups, the estimated times to motor diagnosis were $>12.8,7.6-$ 12.8 , and $<7.6$ years, respectively. Four groups were defined in this analysis: control, low, medium, and high. For more details, please refer to the study by Downing et al. ${ }^{2}$

\section{Measures}

The full WHODAS includes 36 items in six domains: understanding and communicating; getting around; self-care; getting along with others; activities at home, work, and school; and participation in society. Each item has five response categories $(1=$ none; $2=$ mild; $3=$ moderate; $4=$ severe; $5=$ extreme/cannot do). The 12 -item WHODAS includes two items from each domain, with a total score computed as the sum of the 12 items. $^{1}$ To compare the TFC and WHODAS, the TFC was scaled as TFC loss so that higher scores indicate worse function for all outcomes.

\section{Statistical analysis}

Analysis 1: To examine group differences in baseline status and longitudinal change, participant and companion ratings were analyzed separately using linear mixed effects regression (LMER). ${ }^{5}$ Three nested models were fitted for each outcome and compared by Akaike's information criterion. Analysis 2: To test whether longitudinal changes of participant and companion ratings were equal within each group, participant and companion data were analyzed simultaneously. Sixteen possible candidate models were fitted and a model averaging method ${ }^{6}$ was applied to find reliable slope discrepancies. Fitted curves were

${ }^{1}$ Department of Psychiatry, Carver College of Medicine, The University of lowa, lowa City, IA, USA; ${ }^{2}$ Department of Biostatistics, College of Public Health, The University of lowa, Iowa City, IA, USA; ${ }^{3}$ College of Nursing, The University of lowa, lowa City, IA, USA; ${ }^{4}$ Department of Neurology, Carver College of Medicine, The University of lowa, lowa City, IA, USA; ${ }^{5}$ Department of Psychology, The University of lowa, lowa City, IA, USA

${ }^{6}$ The Huntington Study Group members are listed before references.

*Correspondence: Dr JS Paulsen, The University of lowa Roy J. and Lucille A. Carver College of Medicine, Psychiatry Research, 1-305 Medical Education Building, Iowa City, IA 52242-1000, USA. Tel: +319 353 4551; Fax: +319 353 3003; E-mail: predict-publications@uiowa.edu

Received 9 June 2014; revised 5 December 2014; accepted 25 December 2014; published online 4 March 2015 
drawn using model-averaged parameters over all models. Analysis 3: To evaluate the sensitivity of the 12-item WHODAS, comparison was made to the TFC and the 36-item WHODAS. For each scaled outcome, the model with baseline and longitudinal group effects was fitted and effect sizes were compared among the three outcomes. In all analyses, models were adjusted for gender, years of education, and age at entry.

\section{RESULTS}

Progression group differences (Aim 1)

Table 1 shows the best model for each outcome, and the estimated baseline and longitudinal differences between controls and each progression group. Participants showed baseline group differences only, whereas companions showed both baseline and longitudinal group differences. Compared with controls, participants in the medium and high progression groups reported significantly worse functioning at baseline, and companions of those in the high group reported significantly worse functioning at baseline. In contrast, only companions of those in the medium and high groups reported significantly worse functional decline over time.
Self- $v s$ informant-rated WHODAS (Aim 2)

Figure 1 shows the fitted curves for participant and companion outcomes (analyzed simultaneously). The participant (dark blue) and companion (light blue) curves were relatively similar in each group from the control to the medium group, indicating similar rates of change over time in participant and companion ratings. In contrast, there was a divergence between the two curves in the high group, indicating companions reported worse functional decline over time than participants. The divergence in the high group was statistically more reliable than the other groups.

\section{Sensitivity of 12-item WHODAS (Aim 3)}

We compared the companion 12-item WHODAS total to the TFC and companion 36-item WHODAS total, focusing on companions because they better detected longitudinal change than participants. ${ }^{2}$ Figure 2 shows fitted curves for the scaled WHODAS and TFC. At baseline, the 12-item WHODAS $(z=3.55)$ had a larger difference than TFC loss $(z=2.33)$ but a smaller difference than the 36-item WHODAS $(z=3.68)$ in the high group. For longitudinal changes, the 12-item WHODAS $(z=2.45)$ showed a larger difference than both the 36 -item

Table 1 Comparison of World Health Organization Disability Assessment Schedule (WHODAS) 12-item scores between the control group and each gene expanded group for the best models

Group differences relative to controls

Baseline differences Longitudinal differences

\begin{tabular}{|c|c|c|c|c|c|c|}
\hline Measure (best model) & Low est. (SE) & Med est. (SE) & High est. (SE) & Low est. (SE) & Med est. (SE) & High est. (SE) \\
\hline inont 12 itom Tot & $0.031(0.106)$ & $0.224(0.092)^{*}$ & $0.309(0.087)^{* * *}$ & & & \\
\hline ompanion 12-item Total (baseline+longitudinal group & $0.187(0.106)$ & $0.099(0.094)$ & $0.301(0.091)^{* * *}$ & $-0.057(0.099)$ & $0.268(0.091)^{* *}$ & $0.269(0.085)^{* *}$ \\
\hline
\end{tabular}

effect model)

Abbreviations: Est., estimate; SE, standard error; Med, medium. Low, Medium, and High groups indicate low, medium, and high estimated probability of motor diagnosis in 5 years, respectively. ${ }^{* * *} P<0.001,{ }^{* *} P<0.01,{ }^{*} P<0.05$

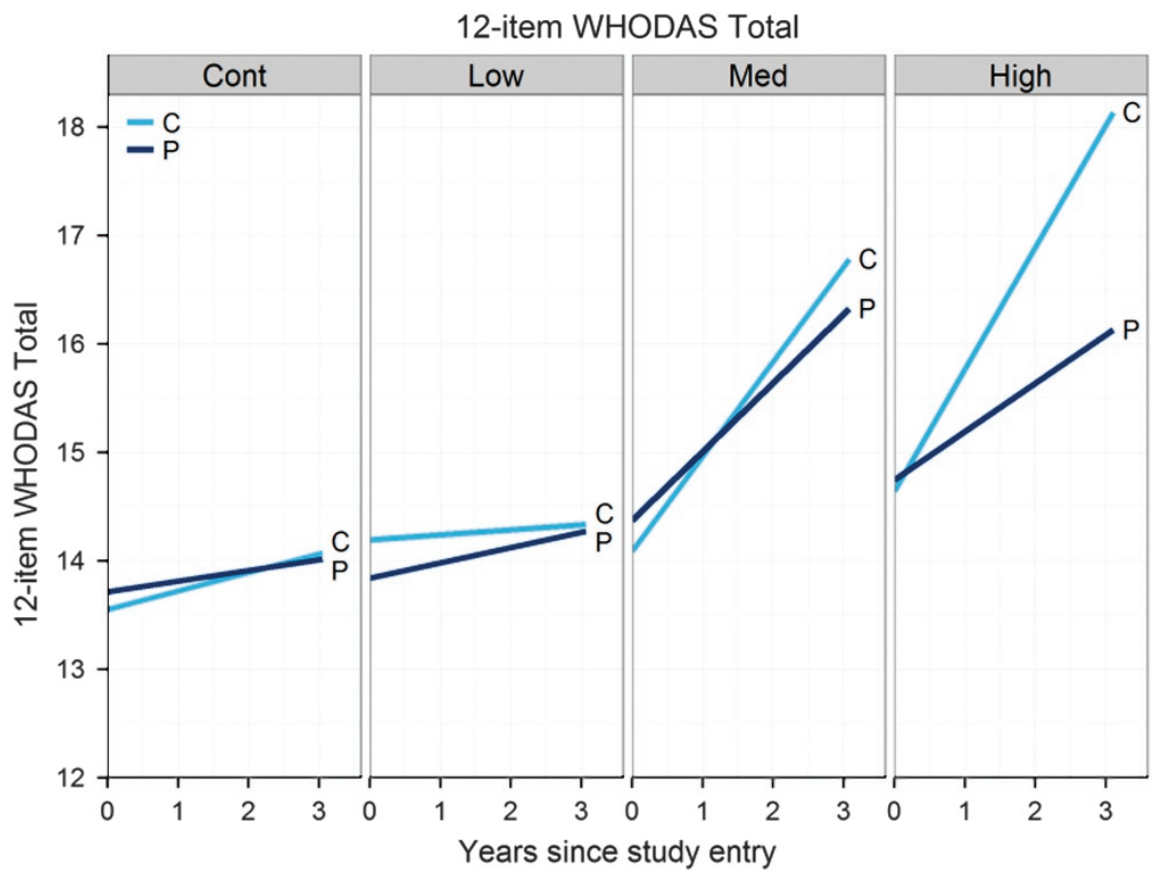

Figure 1 Fitted linear mixed effects regression curves by group for participant $(P)$ and companion $(C)$ ratings of the 12-item World Health Organization Disability Assessment Schedule (WHODAS) 2.0. 

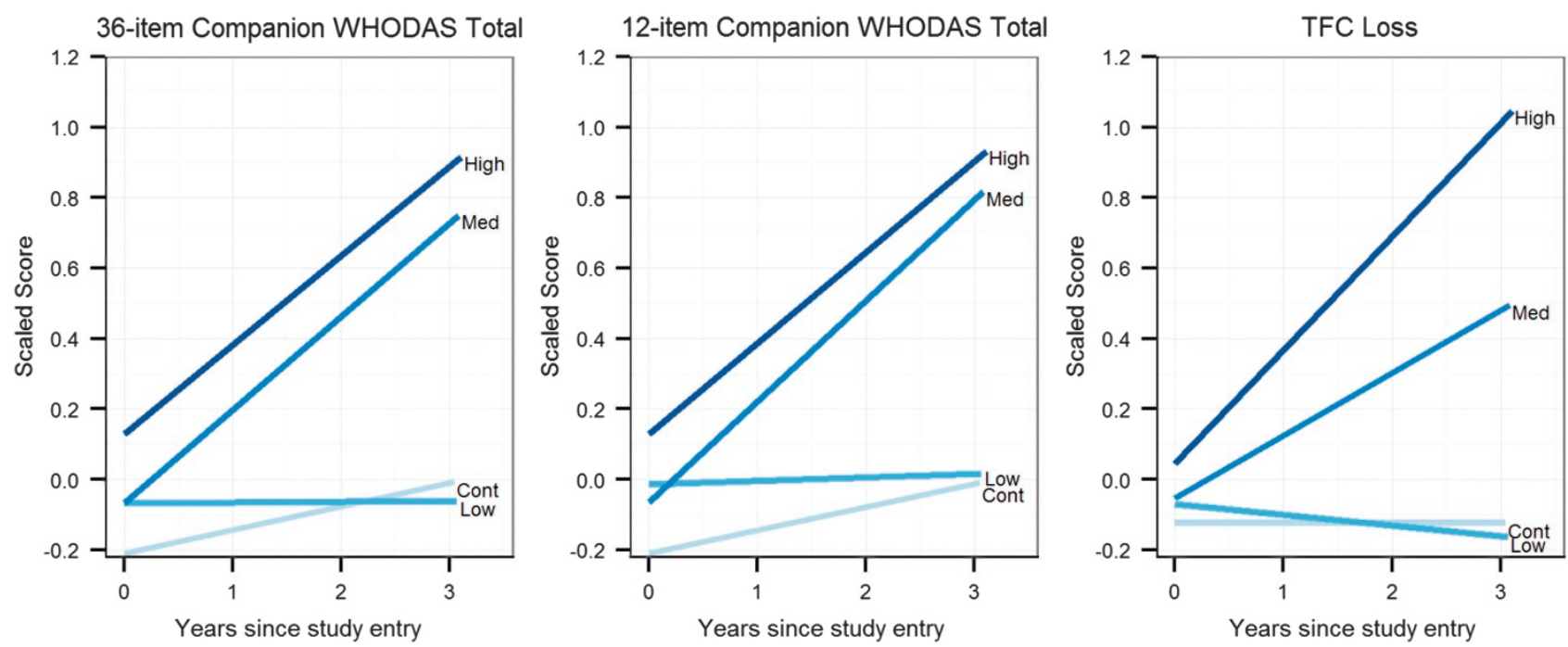

Figure 2 Fitted linear mixed effects regression curves by group for scaled companion 36-item World Health Organization Disability Assessment Schedule (WHODAS) 2.0, companion 12-item WHODAS 2.0, and Total Functional Capacity (TFC) loss scores.

WHODAS $(z=2.35)$ and TFC loss $(z=1.93)$ in the medium group. The 12-item WHODAS $(z=2.27)$ showed a smaller difference than the 36-item WHODAS $(z=2.33)$ and TFC loss $(z=3.76)$ in the high group.

\section{DISCUSSION}

This is the first study that we know of that has undertaken a longitudinal analysis of the 12-item WHODAS 2.0 in a prodromal HD sample. Both participant and companion ratings showed baseline differences by disease progression group, whereas only companion ratings showed longitudinal change differences by disease progression group. To detect differences in baseline levels and longitudinal change by progression group, the 12-item WHODAS seems to be a useful daily function measure in prodromal HD and may be useful for future clinical trials in HD. Companions reported a faster decline of daily function over time than participants in the high group, suggesting participants might experience declining self-awareness of functional changes owing to frontal-subcortical dysfunction, which occurs in HD. ${ }^{7}$ This result indicates proxy measures may be more reliable than self-reported ones in later stages of prodromal HD disease progression, and supports the DSM-5 recommendation of using proxy measures when individuals have impaired cognitive function. Our findings with the 12-item WHODAS were consistent with the previous longitudinal analysis of the 36-item WHODAS. ${ }^{2}$

For individuals with prodromal HD, it has been challenging to find sensitive daily function measures that can detect longitudinal change. ${ }^{8}$ When we assessed the ability of the 12-item WHODAS 2.0 in comparison with the TFC and the 36-item WHODAS 2.0, the short version of the companion WHODAS detected more reliable functional decline over time than the full version and TFC in the medium group. The 12-item WHODAS was also able to detect longitudinal change in the high group and had similar functional decline over time compared with the 36-item WHODAS. In addition, only $10 \%$ of respondents showed longitudinal change for the TFC unlike the 12-item WHODAS, where $68 \%$ showed change over time. Therefore, the 12 -item WHODAS seems to have better sensitivity in detecting longitudinal change in an earlier stage of prodromal HD than the TFC and the 36item WHODAS. The current study presents evidence that the 12-item WHODAS 2.0 has similar psychometric properties to the full version.
This suggests the importance of selecting reliable items for a reduced measure. In a separate analysis (unpublished), people with geneexpansion showed longitudinal differences relative to controls in three subscales - understanding, getting along, and life activities. Using the largest sample of participants with prodromal HD to date, our findings suggest the short version can be used as a general index of HD disease progression. The 12-item version was better able to detect longitudinal change in daily functioning in the earlier stages of prodromal HD than the TFC and the 36-item WHODAS. The improved detection of functional decline and the briefer administration time of the short version suggest it might be a useful part of a clinical trial battery to test the ability of new treatments and interventions to preserve daily function in prodromal HD. The effect of individual items on the performance of the 12-item WHODAS total is an area of future research.

\section{CONFLICT OF INTEREST}

Dr Paulsen and Dr Williams' work has been funded by the National Institutes of Health. Dr Kim, Dr Long, Mr Mills and Dr Downing declare no potential conflicts of interest.

\section{ACKNOWLEDGEMENTS}

We thank the PREDICT-HD sites, the study participants, the National Research Roster for Huntington Disease Patients and Families, the Huntington's Disease Society of America and the Huntington Study Group. This publication was supported by the National Center for Advancing Translational Sciences, and the National Institutes of Health (NIH), through Grant 2 UL1 TR000442-06.

The content is solely the responsibility of the authors and does not necessarily represent the official views of the NIH. This research is supported by the National Institutes of Health, National Institute of Neurological Disorders and Stroke (5R01NS040068) awarded to Dr Paulsen, CHDI Foundation, Inc (A3917) awarded to Dr Paulsen, Cognitive and Functional Brain Changes in Preclinical Huntington's Disease (HD) (5R01NS054893) awarded to Dr Paulsen, 4D Shape Analysis for Modeling Spatiotemporal Change Trajectories in Huntington's (1U01NS082086), Functional Connectivity in Premanifest Huntington's Disease (1U01NS082083), and Basal Ganglia Shape Analysis and Circuitry in Huntington's Disease (1U01NS082085). 


\section{PREDICT-HD INVESTIGATORS, COORDINATORS, MOTOR} RATERS, COGNITIVE RATERS

Stephen Cross, Patricia Ryan, Isabella De Soriano, Courtney Shadrick, Amanda

Miller, and Eric A. Epping (University of Iowa, Iowa City, Iowa, USA).

Edmond Chiu, Joy Preston, Anita Goh, Stephanie Antonopoulos, and

Samantha Loi (St Vincent's Hospital, The University of Melbourne, Kew,

Victoria, Australia).

Phyllis Chua, and Angela Komiti (The University of Melbourne, Royal

Melbourne Hospital, Melbourne, Australia).

Lynn Raymond, Joji Decolongon, Mannie Fan, and Allison Coleman

(University of British Columbia, Vancouver, British Columbia, Canada).

Christopher A. Ross, Mark Varvaris, Maryjane Ong, and Nadine Yoritomo

(Johns Hopkins University, Baltimore, Maryland, USA).

William M. Mallonee and Greg Suter (Hereditary Neurological Disease

Centre, Wichita, Kansas, USA).

Ali Samii, Emily P. Freney, and Alma Macaraeg (University of Washington

and VA Puget Sound Health Care System, Seattle, Washington, USA).

Randi Jones, Cathy Wood-Siverio, and Stewart A. Factor (Emory University

School of Medicine, Atlanta, Georgia, USA).

Roger A. Barker, Sarah Mason, and Natalie Valle Guzman (John van Geest

Centre for Brain Repair, Cambridge, UK).

Elizabeth McCusker, Jane Griffith, Clement Loy, Jillian McMillan and David

Gunn (Westmead Hospital, Sydney, Australia).

Michael Orth, Sigurd Sü $\beta$ muth, Katrin Barth, Sonja Trautmann, Daniela

Schwenk, and Carolin Eschenbach (University of Ulm, Ulm, Germany).

Kimberly Quaid, Melissa Wesson, and Joanne Wojcieszek (Indiana

University School of Medicine, Indianapolis, IN, USA).

Mark Guttman, Alanna Sheinberg, Albie Law, and Irita Karmalkar (Centre

for Addiction and Mental Health, University of Toronto, Markham, Ontario,

Canada).

Susan Perlman and Brian Clemente (UCLA Medical Center, Los Angeles,

California, USA).

Michael D. Geschwind, Sharon Sha, Joseph Winer, and Gabriela Satris

(University of California San Francisco, San Francisco, California, USA).

Tom Warner and Maggie Burrows (National Hospital for Neurology and

Neurosurgery, London, UK).

Anne Rosser, Kathy Price, and Sarah Hunt (Cardiff University, Cardiff,

Wales, UK)

Frederick Marshall, Amy Chesire, Mary Wodarski, and Charlyne Hickey

(University of Rochester, Rochester, New York, USA).

Peter Panegyres, Joseph Lee, Maria Tedesco, and Brenton Maxwell

(Neurosciences Unit, Graylands, Selby-Lemnos \& Special Care Health Services,

Perth, Australia).

Joel Perlmutter, Stacey Barton, and Shineeka Smith (Washington University,

St Louis, Missouri, USA).

Zosia Miedzybrodzka, Daniela Rae, Vivien Vaughan, and Mariella

D’Alessandro (Clinical Genetics Centre, Aberdeen, Scotland, UK).

David Craufurd, Judith Bek, and Elizabeth Howard (University of

Manchester, Manchester, UK).

Pietro Mazzoni, Karen Marder, and Paula Wasserman (Columbia University

Medical Center, New York, New York, USA).

Rajeev Kumar, Diane Erickson, Christina Reeves, and Breanna Nickels

(Colorado Neurological Institute, Englewood, Colorado, USA).

Vicki Wheelock, Lisa Kjer, Amanda Martin, and Sarah Farias (University of

California Davis, Sacramento, California, USA)

Wayne Martin, Oksana Suchowersky, Pamela King, Marguerite Wieler, and

Satwinder Sran (University of Alberta, Edmonton, Alberta, Canada).
Anwar Ahmed, Stephen Rao, Christine Reece, Alex Bura, and Lyla Mourany (Cleveland Clinic Foundation, Cleveland, Ohio, USA).

Executive Committee

Jane S. Paulsen, Principal Investigator, Eric A. Epping, Megan M. Smith,

Jeffrey D. Long, Hans J. Johnson, Jeremy H. Bockholt, and Kelsey Montross Scientific Consultants

Brain: Jean Paul Vonsattell and Carol Moskowitz (Columbia University

Medical Center); Stacie Vik (University of Iowa).

Cognitive: Deborah Harrington (University of California, San Diego);

Tamara Hershey (Washington University); Holly Westervelt (Rhode Island

Hospital/Alpert Medical School of Brown University); Megan M. Smith, and

David J. Moser (University of Iowa).

Functional: Janet Williams and Nancy Downing (University of Iowa).

Imaging: Hans J. Johnson (University of Iowa); Elizabeth Aylward (Seattle

Children's Research Institute); Christopher A. Ross (Johns Hopkins

University); Vincent A. Magnotta (University of Iowa); and Stephen Rao

(Cleveland Clinic, Cleveland, OH.).

Psychiatric: Eric A. Epping (University of Iowa); David Craufurd (University of Manchester).

Core Sections

Biostatistics: Jeffrey D. Long, Ji-In Kim, James A. Mills, Ying Zhang, Dawei Liu, Wenjing Lu, and Spencer Lourens (University of Iowa).

Ethics: Cheryl Erwin (Texas Tech University Health Sciences Center); Eric A. Epping and Janet Williams (University of Iowa); Martha Nance (University of Minnesota).

Biomedical Informatics: H. Jeremy Bockholt and Ryan Wyse (University of Iowa).

1 Ustun TB, Chatterji S, Kostanjsek $\mathrm{N}$ et al: Developing the World Health Organization Disability Assessment Schedule 2.0. Bull World Health Organ 2010; 88: 815-823.

2 Downing NR, Kim JI, Williams JK et al: WHODAS 2.0 in prodromal Huntington disease: measures of functioning in neuropsychiatric disease. Eur J Hum Genet 2014, 22: 958-963.

3 Paulsen JS, Hayden M, Stout JC et al: Preparing for preventive clinical trials: the PredictHD study. Arch Neurol 2006; 63: 883-890.

4 Zhang Y, Long JD, Mills JA, Warner JH, Lu WJ, Paulsen JS: Indexing disease progression at study entry with individuals at-risk for Huntington disease. Am J Med Genet B Neuropsychiatr Genet 2011; 156B: 751-763.

5 Verbeke G, Molenberghs G: Linear mixed models for longitudinal data. New York: Springer, 2000.

6 Burnham KP, Anderson DR: Model Selection and Multimodel Inference: a Practical Information-theoretic Approach, 2nd edn. New York: Springer, 2002.

7 Duff K, Paulsen JS, Beglinger LJ et al: "Frontal" behaviors before the diagnosis of Huntington's disease and their relationship to markers of disease progression: evidence of early lack of awareness. J Neuropsychiatry Clin Neurosci 2010; 22: 196-207.

8 Paulsen JS, Wang C, Duff $\mathrm{K}$ et al: Challenges assessing clinical endpoints in early Huntington disease. Mov Disord 2010; 25: 2595-2603.

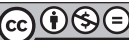

This work is licensed under a Creative Commons Attribution-NonCommercial-NoDerivs 3.0 Unported License. The images or other third party material in this article are included in the article's Creative Commons license, unless indicated otherwise in the credit line; if the material is not included under the Creative Commons license, users will need to obtain permission from the license holder to reproduce the material. To view a copy of this license, visit http://creativecommons.org/licenses/by-nc-nd/3.0/ 